\title{
Off-energy-shell continuation of the nucleon-nucleon and meson-nucleon transition matrices in the presence of inelasticities*
}

\author{
P. U. Sauer ${ }^{\dagger}$ and Alpar Sevgen ${ }^{\ddagger}$ \\ Laboratory for Nuclear Science and Department of Physics, Massachusetts Institute of Technology, Cambridge, Massachusetts 02139
}

(Received 30 June 1975)

\begin{abstract}
A technique for constructing the nucleon-nucleon transition matrices $T$ in the presence of inelastic channels is given. The present technique avoids the usual potential fit and continues $T$ off the energy shell directly consistent with the two-body scattering data and consistent with the properties of possible two-body bound states. The respective $T$ matrices are determined by a real symmetric matrix function $\left\langle E_{1} \alpha_{1}|\sigma| E_{2} \alpha_{2}\right\rangle$ of the continuous energy variable $E$ and the discrete eigenchannel label $\alpha$. The on-shell part of $\sigma$ is given by the eigenphases; the arbitrariness of $T$ is the off-shell continuation of the phase-shift matrix $\left\langle E \alpha_{1}|\sigma| E \alpha_{2}\right\rangle$ into two dimensions. The present technique of constructing $T$ makes the experimental nucleon-nucleon and mesonnucleon scattering data readily available as input for nuclear theory extended to include baryon resonances.
\end{abstract} $\left[\begin{array}{c}\text { NUCLEAR STRUCTURE nucleon-nucleon and meson-nucleon interactions, on } \\ \text { shell and off shell with inelasticities. }\end{array}\right]$

\section{INTRODUCTION}

A Hermitian potential between nucleons in the baryon ground state has been the standard description of the nucleon-nucleon (NN) interaction ${ }^{1}$ for the purposes of microscopic nuclear-structure calculations. Such a description neglects the existence of inelastic interaction channels which at high enough scattering energies open and in which at least one nucleon is in a baryon resonance state, e.g., is a $\Delta(1236)$, or (and) in which free mesons are created. Neglecting the inelastic channels in NN scattering has usually been considered a safe approximation when accounting for the properties of many-nucleon systems at low energies, a belief which appears now shaken in the light of the neutron-matter results of Ref. 2. Thus, there is evidence that calls for an extension of ordinary realistic NN potentials to inelastic channels-even in the case of low-energy nuclear phenomena. The necessity of a corresponding inclusion of inelasticities in the meson-nucleon (MN) interactions, e.g., of a pion plus baryon resonance channel in the pion-nucleon interaction ${ }^{3}$ or of a pion- $\Sigma$ channel in the kaon-nucleon interaction ${ }^{4}$ is well recognized. The present paper suggests the direct off-shell continuation of the respective two-body transition matrices $T$ as a convenient tool of accomplishing this aim for the $\mathrm{NN}$ and MN interactions.

The phenomenological technique of parametrizing $T$ off shell, previously used ${ }^{5,6}$ in the elastic NN channels only, is generalized to the presence of inelasticities. Only the inelastic channels with two-body fragmentations will be considered. The lifetime of the fragments is assumed infinite. The difference in the channel threshold energies is properly taken into account. The technique of constructing $T$ solves the inverse scattering problem exactly and is in this sense a realistic description of the $\mathrm{NN}$ and $\mathrm{MN}$ interactions in the elastic and inelastic scattering domains. It is therefore well suited to digest the expected wealth of highprecision nucleon-nucleon and pion-nucleon scattering data produced by the new generation of accelerators. It will make these experimental data readily usable as input to nuclear theory extended to include baryon resonances. The $T$ matrices are employed rather than the corresponding potentials in order to avoid the ordinary potential fit. Such a fit is already cumbersome when inelasticities are neglected; it presumably constitutes a formidable task when inelasticities are taken into account. The direct construction of $T$ therefore appears to offer a welcome economical advantage.

The construction of the NN and MN transition matrices requires a phenomenological assumption on the off-shell behavior of $T$. The present general technique is therefore more flexible than the simple separable models suggested in Refs. 3 and 4. The off-shell part of $T$ has to be parametrized, since the fundamental $\mathrm{NN}$ and $\mathrm{MN}$ interactions are theoretically poorly known and scattering experiments can only yield information about the onshell values of the respective transition matrices. In contrast, the properties of many-body systems, e.g., nuclear observables as binding energies, radii, electromagnetic form factors and spectra, 
and the meson-nucleus optical potentials, depend upon the experimentally undetermined and theoretically almost unknown off-shell behavior of $T$. Ideally, one would therefore like to derive the basic interactions, on shell and off shell, from field theory first before proceeding to the study of many-body systems. In practice, however, the hadronic force problem is discouragingly involved and, though important progress has recently been made $^{7,8}$ for the NN interaction, its complete solution is still in the very far future. This frustrating situation, of course, should not prevent attempts to understand the properties of many-body systems in terms of the fundamental interactions between their constituents even though the interaction has to be parametrized phenomenologically. Thus we feel that in light of nuclear-structure applications the present phenomenological approach to the hadronic forces is a worthwhile alternative as long as the force problem remains unsolved in a more fundamental and therefore more satisfying way.

The present work generalizes the theoretical methods ${ }^{5,9,10}$ that have been developed for elastic scattering to inelasticities. The fact, that the threshold energies of inelastic channels, in general, are different renders the coupled-channel problem of inelasticities essentially different from the coupled-channel problem arising from tensor forces. ${ }^{10}$ This is one important reason for the present account of the extended theory. This difference in threshold energies makes the relative two-body momentum channel-dependent. The use of an energy representation $\{|E c\rangle\}$ for the $T$ matrices, in contrast to the momentum representation previously used, is therefore required. The energy representation will also easily allow one to introduce corrections to the nonrelativistic kinetic energies in the spirit of "minimal relativity." Section II defines the complete set of basis states $\{|E c\rangle\}$ of any channel $c$ and gives some other useful definitions involving the $T$ matrix and the corresponding multichannel scattering wave functions. The transition matrix is then continued off the energy shell in Sec. III for the case in which the multichannel scattering states form a complete set. The possible presence of a bound state and the possibility of a hard core interaction are neglected in Sec. III in order to present clearly the modifications of the previous work necessary because of inelasticities. The problem of the completeness defect of the scattering states due to a possible bound state or (and) a possible hard core interaction is then taken up in Sec. IV. There, the off-shell extension of the $T$ matrix is studied for this more general case. In Sec. $V$ we indicate how the presented formalism will be used in practice.

\section{DEFINITIONS}

The inelastic channels considered here for the NN and MN scattering process are those with twobody fragmentations. For our purposes, a channel is defined as a set of two-body states with definite quantum numbers, some being conserved, the others not being conserved, in the scattering process. The two-body interaction is assumed to conserve total angular momentum $j$, parity $p$, isospin $t$, and isospin projection $m_{t}$. It is independent of the angular-momentum projection $m_{j}$. However, the two-body interaction is allowed to mix orbital momenta $l$, the two-body spin $s$, and different partitions $\zeta$ of the two-body system with the two fragment masses $m_{\zeta}(1)$ and $m_{\zeta}(2)$. The set of conserved quantum numbers $\left(j m_{j} p t m_{t}\right)$ is denoted by $Q$, the set of nonconserved quantum numbers $(l s \zeta)$ by $c$. The channels with common conserved quantum numbers $Q$ are coupled by the two-body interaction; they form the partial wave $Q$.

The two-body transition matrix in the c.m. system $\left\langle E_{1} \hat{k}_{1}|\boldsymbol{T}(\omega)| E_{2} \hat{k}_{2}\right\rangle$ is an operator in channel space and a function of the relative energies and the directions of relative momenta before the scattering process, i.e., $E_{2}$ and the unit vector $\hat{k}_{2}$ and after it, i.e., $E_{1}$ and $\hat{k}_{1}$. The energy available for the scattering process is $\omega$. The terminology "on shell," "half shell," and "off shell" is the same as in Ref. 5. The channel decomposition of $\left\langle E_{1} \hat{k}_{1}|T(\omega)| E_{2} \hat{k}_{2}\right\rangle$ is

$$
\begin{aligned}
& \left\langle E_{1} \hat{k}_{1}|T(\omega)| E_{2} \hat{k}_{2}\right\rangle \\
& =\sum_{Q} \sum_{c_{1} c_{2}} i^{l_{2}-l_{1}} \Lambda_{c_{1} Q}\left(\hat{k}_{1}\right)\left\langle E_{1} c_{1} Q|T(\omega)| E_{2} c_{2} Q\right\rangle \Lambda_{c_{2} Q}^{\dagger}\left(\hat{k}_{2}\right) .
\end{aligned}
$$

The $\Lambda_{c}(\hat{k})$ are the surface functions which account for the angular and isospin dependence of the channels and which specify their type of fragmentation, i.e.,

$$
\Lambda_{c Q}(k)=\sum_{m_{l} m_{s}}\left\langle l m_{l} s m_{s} \mid j m_{j}\right\rangle Y_{l m_{l}}(\hat{k})\left|s m_{s}\right\rangle\left|t m_{t}\right\rangle|\zeta\rangle
$$

The vector $\left|s m_{s}\right\rangle\left(\left|t m_{t}\right\rangle\right)$ denotes the two-body spin (isospin) state. The vector $|\zeta\rangle$ differentiates between the various two-body partitions. The representation

$$
\left\langle\overrightarrow{\mathbf{r}} \mid E c_{2} Q_{2}\right\rangle=\sum_{c_{1} Q_{1}} \Lambda_{c_{1} Q_{1}}(\hat{r})\left\langle r c_{1} Q_{1} \mid E c_{2} Q_{2}\right\rangle
$$

with the radial channel functions

$$
\left\langle r c_{1} Q_{1} \mid E c_{2} Q_{2}\right\rangle=\delta_{c_{1} c_{2}} \delta Q_{1} Q_{2}\left[\frac{2}{\pi} \rho_{c_{2}}(E)\right]^{1 / 2} r j_{l_{2}}\left(k_{\zeta_{2}} r\right)
$$


is used. It ensures the simple volume elements $d r d \hat{r}$ and $d E d \hat{k}$ in the integrations. The relation between the energy $E$ in the c.m. system and the magnitude of the relative momentum $k_{\zeta}$ is channel-dependent. The theory described in the following is independent of its particular form. Thus, the relativistically correct relation

$$
\begin{aligned}
E= & \left\{\left[m_{\zeta}(1) c^{2}\right]^{2}+\hbar^{2} k_{\zeta}^{2} c^{2}\right\}^{1 / 2} \\
& +\left\{\left[m_{\zeta}(2) c^{2}\right]^{2}+\hbar^{2} k_{\zeta}^{2} c^{2}\right\}^{1 / 2}
\end{aligned}
$$

can be used for $\mathrm{NN}$ and $\mathrm{MN}$ scattering. The density of states with fragmentation $\zeta, \rho_{c}(E)$, is $k_{\zeta}^{2} d k_{\zeta} / d E$. The radial channel functions $\left\langle r c_{1} Q \mid E c_{2} Q\right\rangle$ of Eq. (4) are defined for all energies larger than the threshold energy $\epsilon_{c_{2}}=\left[m_{\zeta_{2}}(1) c^{2}+m_{\zeta_{2}}(2) c^{2}\right]^{1 / 2}$. They are orthogonal and complete in each partial wave $Q$. The two-body potential $V$ has the same channel decomposition as the transition matrix $T(\omega)$. The $T$ matrix and the potential $V$ are matrices as far as their dependence on the coupled channels $c$ is concerned. They factorize according to partial waves. Since we treat each partial wave separately, the partial wave label $Q$ will be dropped from now on.

The scattering states with outgoing waves are obtained from the potential $V$ by means of the Lippmann-Schwinger equation

$$
\left|\psi^{+}(E) c\right\rangle=|E c\rangle+\left(E-H^{0}+i 0\right)^{-1} V\left|\psi^{+}(E) c\right\rangle .
$$

In Eq. (6) $H^{0}$ contains the kinetic energy operator of relative motion and the rest energy of the channels normalized such that the lowest threshold energy $\epsilon_{c}$ coincides with the zero of the chosen energy scale. The usual identity between $T$ matrix, potential, and scattering wave function

$$
T(E+i 0)|E c\rangle=V\left|\psi^{+}(E) c\right\rangle
$$

yields, together with Eq. (6), a variety of relations between the channel-projected properties:

$$
\begin{aligned}
& \left\langle E_{1} c_{1}\left|T\left(E_{2}+i 0\right)\right| E_{2} c_{2}\right\rangle=\sum_{c} \int_{\epsilon_{c}}^{\infty} d E\left\langle E_{1} c_{1}|V| E c\right\rangle\left\langle E c \mid \psi^{+}\left(E_{2}\right) c_{2}\right\rangle, \\
& \left\langle E_{1} c_{1} \mid \psi^{+}\left(E_{2}\right) c_{2}\right\rangle=\delta\left(E_{1}-E_{2}\right) \delta_{c_{1} c_{2}}+\left(E_{2}-E_{1}+i 0\right)^{-1}\left\langle E_{1} c_{1}\left|T\left(E_{2}+i 0\right)\right| E_{2} c_{2}\right\rangle, \\
& \left\langle E_{1} c_{1}|T(\omega)| E_{2} c_{2}\right\rangle=\left\langle E_{1} c_{1}|V| E_{2} c_{2}\right\rangle+\sum_{c} \int_{\epsilon_{c}}^{\infty} d E\left\langle E_{1} c_{1}|V| E c\right\rangle(\omega-E)^{-1}\left\langle E c|T(\omega)| E_{2} c_{2}\right\rangle .
\end{aligned}
$$

Equation (8c) follows from the Lippmann-Schwinger equation for the $T$ matrix.

In the asymptotic region the scattering wave function takes on the form

$$
\left\langle\overrightarrow{\mathbf{r}} \mid \psi^{+}(E) c_{2}\right\rangle \sim-\frac{1}{2 i}\left[g_{c_{2}}(E, r) \Lambda_{c_{2}}(\hat{r})-\sum_{c_{1}} \mathcal{O}_{c_{1}}(E, r) \Lambda_{c_{1}}(\hat{r})\left\langle c_{1}|S(E)| c_{2}\right\rangle\right],
$$

where the sum over $c_{1}$ only includes the channels open at energy $E$ and where $g_{c}(E, r)\left[\theta_{c}(E, r)\right]$ are the asymptotic forms of the incoming [outgoing] waves in channel $c$, i.e.,

$$
g_{c}(E, r)=\Theta_{c}^{*}(E, r)=\left[\frac{2}{\pi} \rho_{c}(E)\right]^{1 / 2} \frac{\exp \left[-i\left(k_{\zeta} r-\frac{1}{2} l_{c} \pi\right)\right]}{k_{\zeta}} \text {. }
$$

The amplitude of the outgoing wave yields the $S$ matrix which is related to the on-shell $T$ matrix by

$$
\left\langle c_{1}|S(E)| c_{2}\right\rangle=\delta_{c_{1} c_{2}}-2 \pi i\left\langle E c_{1}|T(E+i 0)| E c_{2}\right\rangle .
$$

Instead of the basis states $|E c\rangle$ of Eqs. (3) and (4), the eigenchannel states

$$
\begin{aligned}
& \left|E_{2} \alpha\right\rangle=\sum_{c} \int_{\epsilon_{c}}^{\infty} d E_{1}\left|E_{1} c\right\rangle\left\langle E_{1} c \mid E_{2} \alpha\right\rangle, \\
& \left\langle E_{1} c \mid E_{2} \alpha\right\rangle=\delta\left(E_{1}-E_{2}\right)\left\langle c\left|O\left(E_{2}\right)\right| \alpha\right\rangle,
\end{aligned}
$$

will frequently be used. This is the representation which diagonalizes the $S$ matrix. The threshold energies for the eigenchannels and the physical channels are the same, i.e., $\epsilon_{\alpha}=\epsilon_{c}$. The matrix $O(E)$ is real and orthogonal, since the $S$ matrix is unitary and symmetric. As the basis states $|E c\rangle$, the eigenchannel states $|E \alpha\rangle$ are eigenstates of $H^{0}$ and are or thogonal and complete,

$$
\begin{aligned}
& \left\langle E_{1} \alpha_{1} \mid E_{2} \alpha_{2}\right\rangle=\delta\left(E_{1}-E_{2}\right) \delta_{\alpha_{1} \alpha_{2}}, \\
& \sum_{\alpha} \int_{\epsilon_{\alpha}}^{\infty} d E|E \alpha\rangle\langle E \alpha|=1 .
\end{aligned}
$$

In eigenchannel representation the $S$ matrix and the $T$ matrix take on the forms 


$$
\begin{aligned}
& \left\langle\alpha_{1}|S(E)| \alpha_{2}\right\rangle=\delta_{\alpha_{1} \alpha_{2}} \exp \left[2 i \eta\left(E \alpha_{2}\right)\right], \\
& \left\langle E \alpha_{1}|T(E+i 0)| E \alpha_{2}\right\rangle=-(1 / \pi) \delta_{\alpha_{1} \alpha_{2}} \sin \eta\left(E \alpha_{2}\right) \exp \left[i \eta\left(E \alpha_{2}\right)\right],
\end{aligned}
$$

where $\eta(E \alpha)$ is the eigenchannel phase shift. The asymptotic behavior (9) of the coordinate-space scattering wave function is

$$
\left\langle\overrightarrow{\mathbf{r}} \mid \psi^{+}(E) c_{2}\right\rangle \sim \sum_{c_{1}}\left[\frac{2}{\pi} \rho_{c_{1}}(E)\right]^{1 / 2} \Lambda_{c_{1}}(\hat{r}) \sum_{\alpha}\left\langle c_{1}|O(E)| \alpha\right\rangle \frac{\sin \left[k_{\zeta 1} r-\frac{1}{2} l_{1} \pi+\eta(E \alpha)\right]}{k_{\zeta}} \exp [i \eta(E \alpha)]\left\langle\alpha\left|O^{\dagger}(E)\right| c_{2}\right\rangle .
$$

The unitarily transformed scattering wave functions

$$
|\psi(E) \alpha\rangle=\sum_{c}\left|\psi^{+}(E) c\right\rangle\langle c|O(E)| \alpha\rangle \exp [-i \eta(E \alpha)]
$$

remain eigenstates of the full Hamiltonian. They have the representation

$$
\begin{aligned}
\left\langle E_{1} \alpha_{1} \mid \psi\left(E_{2}\right) \alpha_{2}\right\rangle= & \delta\left(E_{1}-E_{2}\right) \delta_{\alpha_{1} \alpha_{2}} \cos \eta\left(E_{2} \alpha_{2}\right) \\
& +\odot\left(E_{2}-E_{1}\right)^{-1}\left\langle E_{1} \alpha_{1}|\phi| E_{2} \alpha_{2}\right\rangle
\end{aligned}
$$

with ${ }^{11}$

$$
\begin{aligned}
& \left\langle E_{1} \alpha_{1}|\phi| E_{2} \alpha_{2}\right\rangle \\
& \quad=\left\langle E_{1} \alpha_{1}\left|T\left(E_{2}+i 0\right)\right| E_{2} \alpha_{2}\right\rangle \exp \left[-i \eta\left(E_{2} \alpha_{2}\right)\right] .
\end{aligned}
$$

The wave functions of Eq. (16) are real. This fact is plausible from Eq. (15), but follows rigorously from the reality ${ }^{11}$ of $\phi$. When using momentumspace wave functions of ordinary normalization, the transformation to real wave functions cannot be achieved as in Eq. (17). We shall frequently refer to $\phi$ as the half-shell $T$ matrix. Its on-shell part is

$$
\left\langle E_{1} \alpha_{1}|\phi| E_{2} \alpha_{2}\right\rangle=-(1 / \pi) \delta_{\alpha_{1} \alpha_{2}} \sin \eta\left(E \alpha_{2}\right) .
$$

Whenever a Hermitian potential exists, which we want to assume, the wave functions of Eq. (16) are orthogonal,

$$
\left\langle\psi\left(E_{1}\right) \alpha_{1} \mid \psi\left(E_{2}\right) \alpha_{2}\right\rangle=\delta\left(E_{1}-E_{2}\right) \delta_{\alpha_{1} \alpha_{2}} .
$$

They are also complete provided there is no bound state and the nature of the interaction does not create an additional completeness defect due to a hard core. If there is a bound states $|B\rangle$ in the partial wave $Q$ considered and if there is an addi tional completeness defect $D$, the completeness relation takes on the form

$$
\sum_{\alpha} \int_{\epsilon_{\alpha}}^{\infty} d E|\psi(E) \alpha\rangle\langle\psi(E) \alpha|+| B\rangle\langle B|+D=1
$$

In Eq. (21) $D$ has the configuration space representation $\left\langle\boldsymbol{r}|D| \boldsymbol{r}^{\prime}\right\rangle=\delta\left(\boldsymbol{r}-\boldsymbol{r}^{\prime}\right) \theta\left(\boldsymbol{r}_{c}-\boldsymbol{r}\right)$, where $\boldsymbol{r}_{c}$ is the radius of the possibly present hard core. The reality of the wave functions $\left\langle E_{1} \alpha_{1} \mid \psi\left(E_{2}\right) \alpha_{2}\right\rangle$ and their orthogonality and completeness relations are the corner stones for the theory presented in the following sections.

\section{OFF-ENERGY SHELL CONTINUATION OF THE MULTICHANNEL $T$ MATRIX WHEN THE SCATTERING STATES ARE COMPLETE}

In this section the multichannel transition matrix is contiuned off the energy shell in the absence of bound states and a hard core component in the interaction. Our aim is to generalize the previously developed techniques ${ }^{5,9,10}$ to those cases where inelastic channels with different thresholds are present and also to include relativistic corrections to the kinetic energy. Hence, to clarify these points we do not consider in this section the complications of unitarity defects. However, most NN and MN partial waves do not contain a bound state, and the assumption of a hard core in either the NN or the MN interaction appears unnecessary. Thus, the results of this section also attain practical value.

The scattering states of Eq. (15), assumed to be complete, are inserted as intermediate states into the relation $T(\omega)=V+V\left(\omega-H^{0}-V\right)^{-1} V$. The unknown Hermitian potential is eliminated in favor of the half-shell $T$. The complete off-shell $T$ matrix is uniquely determined by the half-shell elements:

$$
\begin{aligned}
& \left\langle E_{1} c_{1}|T(\omega)| E_{2} c_{2}\right\rangle=\sum_{\alpha_{1} \alpha_{2}}\left\langle c_{1}\left|O\left(E_{1}\right)\right| \alpha_{1}\right\rangle\left\langle E_{1} \alpha_{1}|T(\omega)| E_{2} \alpha_{2}\right\rangle\left\langle\alpha_{2}\left|O^{\dagger}\left(E_{2}\right)\right| c_{2}\right\rangle \\
& \left\langle E_{1} \alpha_{1}|T(\omega)| E_{2} \alpha_{2}\right\rangle=\left\langle E_{2} \alpha_{1}|\phi| E_{2} \alpha_{2}\right\rangle \cos \eta\left(E_{2} \alpha_{2}\right)+\sum_{\alpha} \int_{\epsilon_{\alpha}}^{\infty} d E\left[(\omega-E)^{-1}-\odot\left(E_{2}-E\right)^{-1}\right]\left\langle E_{1} \alpha_{1}|\phi| E \alpha\right\rangle\left\langle E_{2} \alpha_{2}|\phi| E \alpha\right\rangle .
\end{aligned}
$$


Thus, the arbitrariness in $T$ is the arbitrariness in $\phi$, the half-shell $T$ matrix in eigenchannel representation. Its construction is the main objective of this section.

In the absence of a unitarity defect, the scattering states are orthogonal and complete. Thus, the wave operator

$$
\left\langle E_{1} \alpha_{1}|U| E_{2} \alpha_{2}\right\rangle=\left\langle E_{1} \alpha_{1} \mid \psi\left(E_{2}\right) \alpha_{2}\right\rangle
$$

is real and orthogonal. The singularities of $U$ are given by the phase shifts:

$$
\left\langle E_{1} \alpha_{1}|U| E_{2} \alpha_{2}\right\rangle=\delta_{\alpha_{2} \alpha_{2}}\left\{\delta\left(E_{1}-E_{2}\right) \cos \eta\left(E_{2} \alpha_{2}\right)+\frac{1}{2 \pi} \rho\left(E_{1}-E_{2}\right)^{-1}\left[\sin \eta\left(E_{1} \alpha_{1}\right)+\sin \eta\left(E_{2} \alpha_{2}\right)\right]\right\}+\left\langle E_{1} \alpha_{1}|X| E_{2} \alpha_{2}\right\rangle .
$$

The regular part $X$ is related to the half-shell $T$ matrix $\phi$ :

$$
\begin{aligned}
& \left\langle E_{1} \alpha_{1}|X| E_{2} \alpha_{2}\right\rangle \\
& =\left(E_{2}-E_{1}\right)^{-1}\left[\left\langle E_{1} \alpha_{1}|\phi| E_{2} \alpha_{2}\right\rangle-\frac{1}{2}\left\langle E_{1} \alpha_{1}|\phi| E_{1} \alpha_{2}\right\rangle\right. \\
& \left.\quad-\frac{1}{2}\left\langle E_{2} \alpha_{1}|\phi| E_{2} \alpha_{2}\right\rangle\right] .
\end{aligned}
$$

If one knew the scattering states $\left|\psi^{+}(E) c\right\rangle$, not just their asymptotic behavior, one would know $U$ and from Eq. (17) also the half-shell $T$ matrix. Conversely, any orthogonal operator with singularities as in Eq. (24) corresponds to an acceptable halfshell $T$ matrix with correct on-shell values. We therefore construct half-shell $T$ matrices by constructing orthogonal operators which have the form of Eq. (24). The off-shell arbitrariness in the $T$ matrix is the arbitrariness in the regular part $X$ of these orthogonal operators. From $X$ the half-shell $T$ matrix is obtained:

$$
\begin{aligned}
\left\langle E_{1} \alpha_{1}|\phi| E_{2} \alpha_{2}\right\rangle= & \left(E_{2}-E_{1}\right)\left\langle E_{1} \alpha_{1}|X| E_{2} \alpha_{2}\right\rangle \\
& -\delta_{\alpha_{1} \alpha_{2}}(1 / 2 \pi)\left[\sin \eta\left(E_{1} \alpha_{1}\right)\right. \\
& \left.-\sin \eta\left(E_{2} \alpha_{2}\right)\right] .
\end{aligned}
$$

One possible technique for constructing $T$ calculates the wave operator $U$ of the form Eq. (24) from its antisymmetric part $A$

$$
\left\langle E_{1} \alpha_{1}|A| E_{2} \alpha_{2}\right\rangle=\odot\left(E_{2}-E_{1}\right)^{-1}\left\langle E_{1} \alpha_{1}|\sigma| E_{2} \alpha_{2}\right\rangle,
$$

which contains the symmetric part $\sigma$ of $\phi$, i.e.,

$$
\sigma=\frac{1}{2}\left(\phi+\phi^{\dagger}\right) .
$$

On shell, $\sigma$ reduces to a diagonal matrix in the eigenchannel representation,

$$
\left\langle E \alpha_{1}|\sigma| E \alpha_{2}\right\rangle=-(1 / \pi) \delta_{\alpha_{1} \alpha_{2}} \sin \eta\left(E \alpha_{2}\right) .
$$

The antisymmetric part $A$ determines the complete
$U$ through the unitarity conditions $U^{\dagger} U=U U^{\dagger}=1$. The numerical technique is described in Appendix A.

Since $A$ determines $U$, the half-shell $T$ matrix and also the off-shell $T$ matrix according to Eq. (22) can be calculated from the real matrix function $\left\langle E_{1} \alpha_{1}|\sigma| E_{2} \alpha_{2}\right\rangle$. The function $\sigma$ is symmetric and has the same number of degrees of freedom as the underlying potential. The matrix dimension of $\sigma$ is the number of coupled channels. The onshell elements of $\sigma$ are essentially the phase shifts as given by Eq. (29). The arbitrariness in the $T$ matrix, which is needed off shell in nuclearstructure calculations, consists of the off-shell continuation of this phase shift matrix. The channel mixing matrix $\langle c|O(E)| \alpha\rangle$ does not enter the construction of $U$ at all. It is only needed in the final step Eq. (22a) when the $T$ matrix is transformed back to the representation in terms of physical channels.

\section{OFF-ENERGY-SHELL CONTINUATION OF THE MULTICHANNEL $T$ MATRIX WHEN THE SCATTERING STATES ARE NOT COMPLETE}

We do not know a practical way to construct the wave operator $U$ of Eq. (23) directly in the presence of bound states and other unitarity defects. The real wave operator $U$ is not unitary any longer. In order to apply the methods of constructing $T$, as developed in Sec. III, to this case, we have to rewrite the physical problem by a mathematical trick: We assume a two-particle transition ma$\operatorname{trix} T^{0}$,

$$
T^{0}(\omega)=V^{0}+V^{0}\left(\omega-H^{0}\right)^{-1} T^{0}(\omega),
$$

with the following properties: Its potential $V^{0}$ be given such that (i) it have the same physical channels and eigenchannels, i.e., the same channelmixing matrix $\langle c|O(E)| \alpha\rangle$, (ii) it have the same bound-state energy $E_{B}$ and wave function $\langle E c \mid B\rangle$ as the unknown two-body potential $V$, and (iii) if there are physical reasons demanding a hard core component for the two-body potential $V$, the aux- 
iliary potential $V^{0}$ contain the same such that $\left(V-V^{0}\right) D=0$ holds for the hard core unitarity defect of Eq. (21). The assumptions on $V^{0}$ are quite strong. The Appendix of Ref. 10 discusses a practical way how such an auxiliary potential can be obtained for the physically most interesting unitarity defect of a bound state. The outgoing scattering wave functions of the auxiliary Hamiltonian $H^{0}+V^{0}$, i.e., $\left|\chi^{+}(E) c\right\rangle$, are transformed unitarily, in a way similar to Eq. (17),

$$
\begin{aligned}
\left\langle E_{1} \alpha_{1} \mid \chi\left(E_{2}\right) \alpha_{2}\right\rangle= & \delta\left(E_{1}-E_{2}\right) \delta_{\alpha_{1} \alpha_{2}} \cos \eta^{0}\left(E_{2} \alpha_{2}\right) \\
& +\odot\left(E_{2}-E_{1}\right)^{-1}\left\langle E_{1} \alpha_{1}\left|\phi^{0}\right| E_{2} \alpha_{2}\right\rangle,
\end{aligned}
$$

and can be calculated from the knowledge of $V^{0}$. The eigenchannels of $\left|\chi^{+}(E) c\right\rangle$ and of the physical wave functions are the same by assumption. In contrast, the eigenphases will differ in general, those of $V^{0}$ are denoted by $\eta^{0}(E \alpha)$. The real halfshell $T^{0}$ matrix of $V^{0}$ is $\phi^{0}$; its on-shell part is

$$
\left\langle E \alpha_{1}\left|\phi^{0}\right| E \alpha_{2}\right\rangle=-(1 / \pi) \delta_{\alpha_{1} \alpha_{2}} \sin \eta^{0}\left(E \alpha_{2}\right) .
$$

Since both sets of scattering states, $\left|\psi^{+}(E) c\right\rangle$ and $\left|\chi^{+}(E) c\right\rangle$, have identical completeness defects, the real operator $\left\langle\chi\left(E_{1}\right) \alpha_{1} \mid \psi\left(E_{2}\right) \alpha_{2}\right\rangle$ is orthogonal. The objective is to construct this orthogonal operator using the methods of Sec. III. The physical $T$ matrix will then be obtained from it and from the knowledge of $T^{0}$.

The two-body transition matrix defined by

$$
\begin{aligned}
& T^{1}(\omega)=\left(V-V^{0}\right)+\left(V-V^{0}\right)\left(\omega-H^{0}-V^{0}\right)^{-1} T^{1}(\omega), \\
& T^{1}(\omega)=\left(V-V^{0}\right)+\left(V-V^{0}\right)\left(\omega-H^{0}-V\right)^{-1}\left(V-V^{0}\right)
\end{aligned}
$$

is related to the transition matrices $T(\omega)$ and $T^{0}(\omega)$ by

$$
T(\omega)=T^{0}(\omega)+\Omega^{0}(\stackrel{*}{\omega})^{\dagger} T^{1}(\omega) \Omega^{0}(\omega)
$$

The operator $\Omega^{0}(\omega)$ is defined as

$$
\begin{aligned}
\boldsymbol{\Omega}^{0}(\omega) & =1+\left(\omega-H^{0}\right)^{-1} T^{0}(\omega) \\
& =\left(\omega-H^{0}-V^{0}\right)^{-1}\left(\omega-H^{0}\right) .
\end{aligned}
$$

The relation (34) holds for any two Hermitian potentials $V$ and $V^{0}$ and is derived in Appendix B. We note that because of Eq. (33b) and the properties of $V^{0}$, the projector conditions

$$
\begin{aligned}
& |B\rangle\left\langle B\left|T^{1}(\omega)=T^{1}(\omega)\right| B\right\rangle\langle B|=0, \\
& D T^{1}(\omega)=T^{1}(\omega) D=0
\end{aligned}
$$

are satisfied for the bound state $|B\rangle$ and for a hard core unitarity defect. Thus, the physical transition matrix $T(\omega)$ can be obtained from the knowledge of the matrix elements $\left\langle\chi\left(E_{1}\right) \alpha_{1}\left|T^{1}(\omega)\right| \chi\left(E_{2}\right) \alpha_{2}\right\rangle$ alone using Eq. (34):

$$
\begin{aligned}
\left\langle E_{1} c_{1}|T(\omega)| E_{2} c_{2}\right\rangle= & \sum_{\alpha_{1} \alpha_{2}}\left\langle c_{1}\left|O\left(E_{1}\right)\right| \alpha_{1}\right\rangle\left\langle E_{1} \alpha_{1}|T(\omega)| E_{2} \alpha_{2}\right\rangle\left\langle\alpha_{2}\left|O^{\dagger}\left(E_{2}\right)\right| c_{2}\right\rangle, \\
\left\langle E_{1} \alpha_{1}|T(\dot{\omega})| E_{2} \alpha_{2}\right\rangle= & \left\langle E_{1} \alpha_{1}\left|T^{0}(\omega)\right| E_{2} \alpha_{2}\right\rangle \\
& +\sum_{\alpha_{1}^{\prime} \alpha_{2}^{\prime}} \int_{\epsilon_{\alpha_{1}^{\prime}}}^{\infty} d E_{1}^{\prime} \int_{\epsilon_{\alpha_{2}^{\prime}}}^{\infty} d E_{2}^{\prime}\left\langle\chi\left(E_{1}^{\prime}\right) \alpha_{1}^{\prime}\left|T^{1}(\omega)\right| \chi\left(E_{2}^{\prime}\right) \alpha_{2}^{\prime}\right\rangle\left\langle\chi\left(E_{1}^{\prime}\right) \alpha_{1}^{\prime}\left|\Omega^{0}(\omega)\right| E_{1} \alpha_{1}\right\rangle\left\langle\chi\left(E_{2}^{\prime} \alpha_{2}^{\prime}\right)\left|\Omega^{0}(\omega)\right| E_{2} \alpha_{2}\right\rangle .
\end{aligned}
$$

The matrix elements $\left\langle\chi\left(E_{2}\right) \alpha_{2}\left|\Omega^{0}(\omega)\right| E_{1} \alpha_{1}\right\rangle$ are given in terms of the wave functions (31) according to Eq. (35)

$$
\left\langle\chi\left(E_{2}\right) \alpha_{2}\left|\Omega^{0}(\omega)\right| E_{1} \alpha_{1}\right\rangle=\left(\omega-E_{2}\right)^{-1}\left(\omega-E_{1}\right)\left\langle E_{1} \alpha_{1} \mid \chi\left(E_{2}\right) \alpha_{2}\right\rangle .
$$

The construction of the matrix elements $\left\langle\chi\left(E_{1}\right) \alpha_{1}\left|T^{1}(\omega)\right| \chi\left(E_{2}\right) \alpha_{2}\right\rangle$ is therefore the remaining task.

The physical scattering wave functions $\left|\psi^{+}(E) c\right\rangle$ and those of the auxiliary Hamiltonian are related by the transition matrix $T^{1}(\omega)$ of Eq. (33):

$$
\left|\psi^{+}(E) c\right\rangle=\left[1+\left(E-H^{0}-V^{0}+i 0\right)^{-1} T^{1}(E+i 0)\right]\left|\chi^{+}(E) c\right\rangle .
$$

From Eq. (39), the singularity structure of the real unitary operator $\left\langle\chi\left(E_{1}\right) \alpha_{1} \mid \psi\left(E_{2}\right) \alpha_{2}\right\rangle$ can easily be obtained, 


$$
\left\langle\chi\left(E_{1}\right) \alpha_{1} \mid \psi\left(E_{2}\right) \alpha_{2}\right\rangle=\delta\left(E_{1}-E_{2}\right) \delta_{\alpha_{1} \alpha_{2}} \cos \left[\eta\left(E_{2} \alpha_{2}\right)-\eta^{0}\left(E_{2} \alpha_{2}\right)\right]+\rho\left(E_{2}-E_{1}\right)^{-1}\left\langle\chi\left(E_{1}\right) \alpha_{1}\left|\phi^{1}\right| \chi\left(E_{2}\right) \alpha_{2}\right\rangle,
$$

where $\phi^{1}$ is defined to be

$$
\left\langle\chi\left(E_{1}\right) \alpha_{1}\left|\phi^{1}\right| \chi\left(E_{2}\right) \alpha_{2}\right\rangle=\left\langle\chi\left(E_{1}\right) \alpha_{1}\left|T^{1}\left(E_{2}+i 0\right)\right| \chi\left(E_{2}\right) \alpha_{2}\right\rangle \exp \left\{-i\left[\eta\left(E_{2} \alpha_{2}\right)-\eta^{0}\left(E_{2} \alpha_{2}\right)\right]\right\}
$$

and reduces on shell to

$$
\left\langle\chi(E) \alpha_{1}\left|\phi^{1}\right| \chi(E) \alpha_{2}\right\rangle=-(1 / \pi) \delta_{\alpha_{1} \alpha_{2}} \sin \left[\eta\left(E \alpha_{2}\right)-\eta^{0}\left(E \alpha_{2}\right)\right]
$$

according to Eq. (34). The real unitary operator (40) is identical in form to the physical wave operator of Eq. (17). Only the phase shifts in the onshell elements have to be replaced by the difference in eigenphases of $V$ and $V^{0}$. Thus, the construction procedure for the half-shell $T^{\mathbf{1}}$ matrix in the representation of scattering states of $V^{0}$, i.e., $\phi^{1}$, is identical to the construction procedure of the physical half-shell $T$ matrix $\phi$ in Sec. III. Thus $\phi^{1}$ is determined from its symmetric part $\sigma^{1}$, i.e.,

$$
\begin{aligned}
\left\langle\chi\left(E_{1}\right) \alpha_{1}\left|T^{1}(\omega)\right| \chi\left(E_{2}\right) \alpha_{2}\right\rangle= & \left\langle\chi\left(E_{1}\right) \alpha_{1}\left|\phi^{1}\right| \chi\left(E_{2}\right) \alpha_{2}\right\rangle \cos \left[\eta\left(E_{2} \alpha_{2}\right)-\eta^{0}\left(E_{2} \alpha_{2}\right)\right] \\
& +\sum_{\alpha} \int_{\epsilon_{\alpha}}^{\infty} d E\left[(\omega-E)^{-1}-P\left(E_{2}-E\right)^{-1}\right]\left\langle\chi\left(E_{1}\right) \alpha_{1}\left|\phi^{1}\right| \chi(E) \alpha\right\rangle\left\langle\chi\left(E_{2}\right) \alpha_{2}\left|\phi^{1}\right| \chi(E) \alpha\right\rangle .
\end{aligned}
$$

The on-shell $T$ does not depend on off-shell elements of $\phi^{1}$, and $\phi^{1}$ determines all elements $\left\langle\chi\left(E_{1}\right) \alpha_{1}\left|T^{1}(\omega)\right| \chi\left(E_{2}\right) \alpha_{2}\right\rangle$. The off-shell arbitrariness in the physical two-body $T$ matrix is therefore the arbitrariness in the symmetric part $\sigma^{1}$ of $\phi^{1}$. The matrix $\sigma^{1}$ has the same number of degrees of freedom as the underlying potential. Thus the two-body transition matrix can be constructed consistent with all scattering data even in the presence of bound states and other unitarity defects.

\section{CONCLUSION}

In the case of a multichannel partial wave the two-body transition matrix for NN and MN scattering can be determined from the energy and wave function of a possible bound state, the onshell channel-mixing matrix $O(E)$, and a real symmetric matrix function $\sigma$ or $\sigma^{1}$. The experimental information on the eigenphases $\eta(E \alpha)$ is incorporated in the on-shell elements of $\sigma$ or $\sigma^{1}$; the channel-mixing matrix is needed only to perform the necessary transformation of the two-body transition matrix from eigenchannel representation to the representation of physical channels. The off-shell part of $\sigma$ or $\sigma^{1}$ is the part of the NN and $\mathrm{MN}$ interactions not determined by scattering experiments. This limitation of scattering information requires the off-shell part of $\sigma$ or $\sigma^{1}$ to be parametrized. Otherwise one has to hope for a sudden breakthrough in the hadronic force problem or perform off-shell experiments to determine it.

The construction procedure for the transition matrix $T$ combined with a phenomenological choice of the off-shell $\sigma$ or $\sigma^{1}$ avoids the usual complications of a potential fit. It neatly separates $T$ into elastic and inelastic on-shell information, the only kind of data accessable at present, and unknown off-shell behavior. The two-body $T$ constructed this way can easily be used for microscopic nuclear-structure calculations in the NN case and for determining the meson-nucleus optical potentials in the MN case. It provides an economical link between NN and MN elastic and inelastic scattering data on one side and nuclear theory on the other side. Especially, the direct construction technique for $T$ will make the expected wealth of high-precision NN and MN scattering data readily available for nuclear theory and, on this ground alone, should deserve the attention of experimentalists and theorists alike.

In the region of inelasticities $\mathrm{NN}$ and $\mathrm{MN}$ scattering experiments are presently described ${ }^{12,13}$ in terms of phase shifts for the respective elastic channels $c_{1}$ only. The complex nature of the phase shifts,

$$
\eta\left(E c_{1}\right)=\delta\left(E c_{1}\right)+i \epsilon\left(E c_{1}\right)
$$

accounts for the loss of flux from the elastic channel $c_{1}$ to the open inelastic channels at high enough energies, i.e., 


$$
\begin{aligned}
\left\langle\overrightarrow{\mathrm{r}} \mid \psi^{+}(E) c_{1}\right\rangle \sim & {\left[\frac{2}{\pi} \rho_{c_{1}}(E)\right]^{1 / 2} } \\
& \times \Lambda_{c_{1}}(\hat{\gamma}) \exp \left[i \delta\left(E c_{1}\right)\right] \exp \left[-\epsilon\left(E c_{1}\right)\right] \\
& \times \frac{\sin \left[k_{\zeta_{1}} \gamma-\frac{1}{2} l_{c_{1}} \pi+\delta\left(E c_{1}\right)+i \epsilon\left(E c_{1}\right)\right]}{k_{\zeta_{1}}} .
\end{aligned}
$$

In Eqs. (44) and (45) and in the remainder of this section a possible coupling of NN channels due to the tensor force is neglected for reasons of simplicity in the presentation. The parametrization of the scattering data according to Eq. (44) does not yield the full multichannel $S$ matrix rather its nonunitary part in the direct channel $c_{1}$ only. It therefore appears to be theoretically sufficientand it is also simplest for a start-to replace all inelasticities in NN and MN scattering by one dominant inelastic channel and treat this two-channel problem according to the methods of this paper. The full unitary $S$ matrix in this two-channel case is

$$
S(E)=\left[\begin{array}{cc}
e^{2 i \delta\left(E c_{1}\right)} e^{-2 \epsilon\left(E c_{1}\right)} & i e^{i\left[\delta\left(E c_{1}\right)+\delta\left(E c_{2}\right)\right]}\left[1-e^{\left.-4 \epsilon\left(E c_{1}\right)\right]^{1 / 2}}\right. \\
i e^{i\left[\delta\left(E c_{1}\right)+\delta\left(E c_{2}\right)\right]}\left[1-e^{-4 \epsilon\left(E c_{1}\right)_{1} / 2}\right. & e^{2 i \delta\left(E c_{2}\right)} e^{-2 \epsilon\left(E c_{1}\right)}
\end{array}\right] .
$$

As long as the inelastic channels are not observed directly, but only in their effect on the elastic channel $c_{1}$, the phase shift $\delta\left(E c_{2}\right)$ in the inelastic channel remains experimentally undetermined and has to be parametrized along with the off-shell part of the basic quantity $\sigma$ or $\sigma^{1}$. E.g., in a first primitive description of the $\mathrm{NN}$ interaction one may take $\sigma$ in the elastic channel $c_{1}$ from an ordinary NN potential, ${ }^{6}$ on shell adjusted to the experimental phase shift $\delta\left(E c_{1}\right)$. Assuming that $N \Delta$ production is the dominant source for inelasticity and that $N \Delta$ scattering is dynamically similar to $N N$ scattering on and off shell, the same $\sigma$ may be used in the inelastic channel $c_{2}$ as in $c_{1}$, its energy dependence properly shifted to account for the dif ferent channel threshold, e.g., on shell $\delta\left(E c_{2}\right)$ $=\delta\left(E-\epsilon_{2}, c_{1}\right)$. The coupling part of $\sigma$ may be obtained from the one-pion exchange potential ${ }^{14}$ in Born approximation, the experimental inelasticity parameter is required for the necessary transformation to eigenchannels. Numerical work along these ideas are in progress. The ability to perform nuclear-structure calculations consistent with all available scattering data on the basis interaction between nucleons and between mesons and nucleons appears to make such efforts worthwhile.

\section{APPENDIX A. CONSTRUCTION OF $\phi$ FROM THE KNOWLEDGE OF THE PHASE-SHIFT MATRIX $\sigma$}

Here we assume that the phase-shift matrix $\sigma$ of Eq. (28) is known, i.e., that a choice has been made of its off-shell behavior and of its unknown on-shell phase shifts at high energies or at energies where the experiments are incomplete (see Sec. V). The technique for practically performing the step from $\sigma$ to $\phi$ in the absence of unitarity defects is the subject of this Appendix. The knowledge of $\phi$ enables us then to calculate the off-shell extension of the $T$ matrix according to Eq. (22).

The wave operator $U$ of Eq. (23) splits into a symmetric and an antisymmetric part, i.e., $U=S$ $+A$. The phase-shift matrix $\sigma$ determines the antisymmetric part $A$ of $U$ through Eq. (27), which in turn determines the symmetric part $S$ of $U$ through the unitarity conditions

$$
\begin{aligned}
& S^{2}=1+A^{2}, \\
& S A-A S=0 .
\end{aligned}
$$

The first step in solving Eqs. (A1) is therefore the computation of the matrix elements of $A^{2}$, i.e.,

$$
\left\langle E_{1} \alpha_{1}\left|A^{2}\right| E_{2} \alpha_{2}\right\rangle=-\sum_{\alpha} \int_{\epsilon_{\alpha}}^{\infty} d E\left\langle E_{1} \alpha_{1}|A| E \alpha\right\rangle\left\langle E_{2} \alpha_{2}|A| E \alpha\right\rangle .
$$

The matrix elements of $A$ have principal value singularities. It is desirable to separate out the singularities of the matrix $A$. There is no unique way of doing so, however, that way of separation is chosen which gives well-studied integrals in the computation ${ }^{5}$ of $A^{2}$, when the thresholds are degenerate and relativistic effects are neglected:

$$
\left\langle E_{1} \alpha_{1}|A| E_{2} \alpha_{2}\right\rangle=\rho\left(E_{2}-E_{1}\right)^{-1}\left(E_{2}-\epsilon_{\alpha_{2}}\right)^{-1 / 4}\left(E_{1}-\epsilon_{\alpha_{1}}\right)^{1 / 4}\left\langle E_{1} \alpha_{1}|\sigma| E_{1} \alpha_{2}\right\rangle+\left(E_{2}-\epsilon_{\alpha_{2}}\right)^{-1 / 4}\left\langle E_{1} \alpha_{1}|R| E_{2} \alpha_{2}\right\rangle .
$$

The matrix elements of $R$ are smooth functions of energy,

$$
\left\langle E_{1} \alpha_{1}|R| E_{2} \alpha_{2}\right\rangle=\left(E_{2}-E_{1}\right)^{-1}\left[\left(E_{2}-\epsilon_{\alpha_{2}}\right)^{1 / 4}\left\langle E_{1} \alpha_{1}|\sigma| E_{2} \alpha_{2}\right\rangle-\left(E_{1}-\epsilon_{\alpha_{2}}\right)^{1 / 4}\left\langle E_{1} \alpha_{1}|\sigma| E_{1} \alpha_{2}\right\rangle\right]
$$


The matrix elements of $A^{2}$ can now be computed:

$$
\begin{aligned}
\left\langle E_{1} \alpha_{1}\left|A^{2}\right| E_{2} \alpha_{2}\right\rangle= & -\sum_{\alpha}\left(E_{1}-\epsilon_{\alpha}\right)^{1 / 4}\left(E_{2}-\epsilon_{\alpha}\right)^{1 / 4}\left\langle E_{1} \alpha_{1}|\sigma| E_{1} \alpha\right\rangle\left\langle E_{2} \alpha_{2}|\sigma| E_{2} \alpha\right\rangle \int_{\epsilon_{\alpha}}^{\infty} d E\left(E-\epsilon_{\alpha}\right)^{-1 / 2} \sigma\left(E-E_{1}\right)^{-1}\left(E-E_{2}\right)^{-1} \\
& -\sum_{\alpha}\left(E_{1}-\epsilon_{\alpha}\right)^{1 / 4}\left\langle E_{1} \alpha_{1}|\sigma| E_{1} \alpha\right\rangle \int_{\epsilon_{\alpha}}^{\infty} d E\left(E-\epsilon_{\alpha}\right)^{-1 / 2}\left(E-E_{1}\right)^{-1}\left[\left\langle E_{2} \alpha_{2}|R| E \alpha\right\rangle-\left\langle E_{2} \alpha_{2}|R| E_{1} \alpha\right\rangle\right] \\
& -\sum_{\alpha}\left(E_{2}-\epsilon_{\alpha}\right)^{1 / 4}\left\langle E_{2} \alpha_{2}|\sigma| E_{2} \alpha\right\rangle \int_{\epsilon_{\alpha}}^{\infty} d E\left(E-\epsilon_{\alpha}\right)^{-1 / 2}\left(E-E_{2}\right)^{-1}\left[\left\langle E_{1} \alpha 1|R| E \alpha\right\rangle-\left\langle E_{1} \alpha_{1}|R| E_{2} \alpha\right\rangle\right] \\
& -\sum_{\alpha} \int_{\epsilon_{\alpha}}^{\infty} d E\left(E-\epsilon_{\alpha}\right)^{-1 / 2}\left\langle E_{1} \alpha_{1}|R| E \alpha\right\rangle\left\langle E_{2} \alpha_{2}|R| E \alpha\right\rangle
\end{aligned}
$$

In order to regularize the integrals in the second and third terms of Eq. (A5), the vanishing expression

$$
\int_{\epsilon_{\alpha}}^{\infty} d E\left(E-\epsilon_{\alpha}\right)^{-1 / 2} \odot\left(E-E_{1}\right)^{-1}=0
$$

multiplied with an appropriate constant matrix element is subtracted. The integral in the first term can be evaluated as in Ref. 5 and we obtain

$$
\begin{aligned}
\int_{\epsilon_{\alpha}}^{\infty} d E & \left(E-\epsilon_{\alpha}\right)^{-1 / 2} \odot\left(E-E_{1}\right)^{-1}\left(E-E_{2}\right)^{-1} \\
& =\pi^{2} \delta\left(E_{1}-E_{2}\right)\left(E_{1}-\epsilon_{\alpha}\right)^{-1 / 4}\left(E_{2}-\epsilon_{\alpha}\right)^{-1 / 4} .
\end{aligned}
$$

The factors $\left(E-\epsilon_{\alpha}\right)^{-1 / 2}$ in the other three integrals of (A5) do not create any convergence problems. Using Eq. (29) the operator $A^{2}$ can be rewritten in a compact form

$$
\begin{aligned}
\left\langle E_{1} \alpha_{1}\left|A^{2}\right| E_{2} \alpha_{2}\right\rangle= & -\delta\left(E_{1}-E_{2}\right) \delta_{\alpha_{1} \alpha_{2}} \sin ^{2} \eta\left(E_{2} \alpha_{2}\right) \\
& -\left\langle E_{1} \alpha_{1}|g| E_{2} \alpha_{2}\right\rangle,
\end{aligned}
$$

which also serves as a definition of the matrix $g$ whose elements are smooth functions of energy as can be seen from Eqs. (A5) and (A8). Equation (A5) manifestly shows that $A^{2}$ and $g$ are symmetric operators. All infinite energy integrations in Eq. (A5) and those when Eqs. (A1) are numerically solved are approximated by integrations with a finite number of meshpoints. The operators $g$ and $S$ therefore become finite matrices. Their matrix elements vanish for all energy meshpoints below the threshold energies in either basis state. Using these finite matrices the algorithm of Ref. 5 which solves Eqs. (A1) for $S$ carries over unchanged to the present case and is therefore not repeated here. From $S, \phi$ is obtained according to

$$
\begin{aligned}
\left\langle E_{1} \alpha_{1}|\phi| E_{2} \alpha_{2}\right\rangle= & \left\langle E_{1} \alpha_{1}|\sigma| E_{2} \alpha_{2}\right\rangle \\
& +\left(E_{2}-E_{1}\right)\left\langle E_{1} \alpha_{1}|S| E_{2} \alpha_{2}\right\rangle .
\end{aligned}
$$

The alternative methods for constructing $\phi$ discussed in Ref. 10 can also be used in the present context.

\section{APPENDIX B. TWO POTENTIAL FORMULA FOR THE OFF-SHELL TRANSITION MATRIX}

Equation (34) is proven using identities between the following propagators relevant for the transition matrices $T, T^{0}, T^{1}$ of Sec. IV :

$$
\begin{aligned}
& G(\omega)=\left(\omega-H^{0}-V\right)^{-1}, \\
& G^{0}(\omega)=\left(\omega-H^{0}-V^{0}\right)^{-1}, \\
& g(\omega)=\left(\omega-H^{0}\right)^{-1} .
\end{aligned}
$$

The propagators $G$ and $G^{0}$ satisfy the following relation with the transition matrix $T^{1}$ defined in Eq. (33):

$$
\begin{aligned}
& G(\omega)=G^{0}(\omega)+G^{0}(\omega)\left(V-V^{0}\right) G(\omega), \\
& G(\omega)=G^{0}(\omega)+G^{0}(\omega) T^{1}(\omega) G^{0}(\omega) .
\end{aligned}
$$

Two more relations involving the physical twobody transition matrix $T$ and the auxiliary one, $T^{0}$, follow from (B2b) by specialization

$$
\begin{aligned}
& G(\omega)=g(\omega)+g(\omega) T(\omega) g(\omega), \\
& G^{0}(\omega)=g(\omega)+g(\omega) T^{0}(\omega) g(\omega) .
\end{aligned}
$$

When solving (B3a) for $T$ and using (B2b), one obtains

$$
\begin{aligned}
T(\omega)= & {\left[g^{-1}(\omega) G^{0}(\omega) g^{-1}(\omega)-g^{-1}(\omega)\right] } \\
& +g^{-1}(\omega) G^{0}(\omega) T^{1}(\omega) G^{0}(\omega) g^{-1}(\omega) .
\end{aligned}
$$

The term in square brackets is $T^{0}$ as can be seen from Eq. (B3b). Furthermore, since $\Omega^{0}(\omega)$ defined in Eq. (35) is $G^{0}(\omega) g^{-1}(\omega)$, the Eq. (34) is proven. 
*Work supported in part through funds provided by the U.S. Energy Research and Development Administration under contract No. AT 11-1-3069.

$\dagger$ Present address: Theoretical Physics, Technical University, 3000 Hannover, Germany.

$\ddagger$ Present address: Fizik Bölümü, Bog̃aziçi Üniversitesi, Istanbul, Turkey.

${ }^{1}$ R. V. Reid, Ann. Phys. (N.Y.) 50, 411 (1968); K. Erkelenz, Phys. Lett. 13C, 193 (1974).

${ }^{2}$ A. M. Green and P. Haapakoski, Nucl. Phys. A221, 429 (1974).

${ }^{3}$ J. T. Londergan and E. J. Moniz, Phys. Lett. $\underline{45 B}, 195$ (1973).

${ }^{4}$ M. Alberg, E. M. Henley, and L. Wilets, Phys. Rev. Lett. 30, 255 (1973).

${ }^{5} \mathrm{M}$. Baranger, B. Giraud, S. K. Mukhopadhyay, and P. U. Sauer, Nucl. Phys. A138, 1 (1969).

${ }^{6}$ P. U. Sauer, Ann. Phys. (N.Y.) 80, 242 (1973).

${ }^{7}$ M. Chemtob, J. W. Durso, and D. O. Riska, Nucl. Phys. B38, 141 (1972).

${ }^{8}$ W. N. Cottingham, M. Lacombe, B. Loiseau, J. M. Richard, and R. Vinh Mau, Phys. Lett. 44 B, 1 (1973); Phys. Rev. D 8 , 800 (1973).

${ }^{9}$ M. I. Haftel, Phys. Rev. Lett. 25, 120 (1970).
${ }^{10}$ P. U. Sauer, Nucl. Phys. A170, 497 (1971).

${ }^{11}$ The quantity $\phi$ is an ordinary energy-independent operator in the space of the channel functions $\{|E c\rangle\}$ or $\{|E \alpha\rangle\}, \phi \equiv \int_{0}^{\infty} d E T(E+i 0) \delta\left(E-H^{0}\right) B$, where the operator $B$ can be given in any basis, but has its simplest form in eigenchannel representation, i.e., $\left\langle E_{1} \alpha_{1}|B| E_{2} \alpha_{2}\right\rangle=\delta\left(E_{1}-E_{2}\right) \delta_{\alpha_{1} \alpha_{2}} \exp \left[-i \eta\left(E_{2} \alpha_{2}\right)\right]$. The matrix elements of the operator $\phi$ are real, since $\phi$ satisfies the integral equation

$$
\begin{aligned}
\left\langle E_{1} \alpha_{1}|\phi| E_{2} \alpha_{2}\right\rangle= & \left\langle E_{1} \alpha_{1}|V| E_{2} \alpha_{2}\right\rangle \cos \eta\left(E_{2} \alpha_{2}\right) \\
& +\sum_{\alpha} \int_{\epsilon_{\alpha}}^{\infty} d E\left\langle E_{1} \alpha_{1}|V| E \alpha\right\rangle P\left(E_{2}-E\right)^{-1} \\
& \times\left\langle E \alpha|\phi| E_{2} \alpha_{2}\right\rangle,
\end{aligned}
$$

and the potential matrix elements can be chosen real.

${ }^{12}$ R. A. Arndt, Rev. Mod. Phys. 39, 710 (1967);

U. Amaldi, Jr., ibid. 39, 649 (1967).

${ }^{13}$ D. H. Herndon, A. Barbaro-Galtieri, and A. H. Rosenfeld, LRL Report No. UCRL-20030 $\pi N, 1970$ (unpublished).

${ }^{14} \mathrm{H}$. Sugawara and F. von Hippel, Phys. Rev. $\underline{172}, 1764$ (1968). 